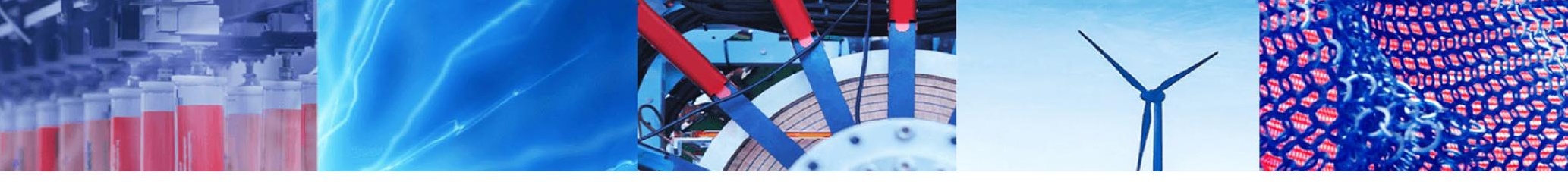

Research Article

\title{
Formation mechanism and compensation methods of profile error in focused ion beam milling of three-dimensional optical microstructures
}

\author{
Xiao Chen ${ }^{1}$ ] $\cdot$ Zhenzhou Ren $^{1} \cdot$ Yan Zhu ${ }^{1} \cdot$ Yixing Wang ${ }^{1} \cdot$ Jianguo Zhang $^{1} \cdot$ Xuefang Wang $^{1} \cdot$ Jianfeng Xu $^{1}$
}

Received: 27 November 2019 / Accepted: 8 March 2020 / Published online: 26 March 2020

(c) Springer Nature Switzerland AG 2020

\begin{abstract}
The formation mechanism and compensation methods of the profile error in focused ion beam (FIB) bitmap milling of three-dimensional (3D) optical microstructure are studied in this paper. The processing parameters such as ion beam currents, dwell times, and beam overlap ratios on the profile error have an optimized parameters combination of 21 $\mathrm{nA}, 10 \mu \mathrm{s}$, and $50 \%$, and the shape accuracy processed under the conditions is $0.44 \mu \mathrm{m}$ of $P-V$ and $0.142 \mu \mathrm{m}$ of RMS, respectively. Inappropriate processing parameters lower either shape accuracy or processing efficiency. By means of an accurate simulation model of the FIB machined 3D profiles, the intrinsic formation mechanism of the profile error can be attributed to ion beam diameters, redeposition effect, and sputter yields, and these factors are inevitable in FIB milling of 3D microstructures. In situ modification and iterative optimization are proposed to compensate the FIB milling error, which both can improve the shape accuracy more than $50 \%$. The in situ modification method needs to load the original bitmap and modified bitmap separately, which is less convenient than the iterative optimization method that only need loading one bitmap file. However, the in situ modification is better than the iterative optimization at decreasing the RMS value of the profile error which indicates the global profiles error of the processed microstructures.
\end{abstract}

Keywords Focused ion beam · Three-dimensional microstructures · Profile error · Formation mechanism · Error compensation

\section{Introduction}

To improve the high quality of imaging or realize some specific functions, traditional optical systems often use the combination of large curved lens, which not only makes the optical system structure complicated and costly, but also occupies larger space. This is extremely detrimental to the optical systems requiring lightweight and miniaturization, such as space detection systems, micro-electromechanical systems (MEMS), and portable medical system or devices. Three-dimensional (3D) microstructural optical components, which consist of 3D micro- or nanoscale structures arranged in a certain order, possess the ability of modulating lights and electromagnetic waves and are able to integrate multiple functions on a thin plane, for example eliminating chromatic aberration, phase difference, and spherical aberration, improving imaging resolution, and increasing field angle [1,2]. Hence, 3D microstructural optical components are a versatile plane optical device to satisfy the demand of lightweight and miniaturization $[3,4]$. Nevertheless, these fine optical devices put forward extremely high request to the manufacturing techniques as the shape accuracy and surface quality of the 3D microstructures have a significant influence on the devices' performance.

Xuefang Wang, memsman@163.com; $\bowtie$ Jianfeng Xu, jfxu@hust.edu.cn | ${ }^{1}$ State Key Laboratory of Digital Manufacturing Equipment and Technology, School of Mechanical Science and Engineering, Huazhong University of Science and Technology, Wuhan 430074, China. 
Over the last decades, different lithographic techniques have been used to fabricate complex 3D microstructures, for instance, photolithography, electron beam lithography (EBL), and nanoimprint lithography [5]. These techniques have their own advantages and disadvantages such as high efficiency, resolution limit, complex steps, costly equipment, and material dependence [5-8]. Focused ion beam (FIB) milling combined with electron column (dualbeam system) a powerful technique for fabricating microand nano-structures (ranging from sub-50 $\mathrm{nm}$ to several tens of microns in size) with a high patterning flexibility on almost any material [9-12]. The technique has the distinctive advantage of direct writing with the ability to control pixel by the ion dose (dwell time), hence permitting to produce 3D patterns with relatively high aspect ratio and without the use of additional masks and resists [13, 14].

According to the material to be machined and the requested geometry, the process needs to set several milling parameters, such as ion currents (ranging from $\mathrm{pA}$ to $\mathrm{nA}$ for $\mathrm{Ga}^{+}$ion source), ion dose (dwell time and number of passes), beam overlap, and pattern strategy (ion beam scan routes) to obtain satisfying results [15]. Vasile et al. [16] have reported a method to machine 3D microstructures by controlling the dwell time at each pixel for milling curved profiles. They milled a concave hemisphere with a diameter of ten microns on a silicon (100) substrate by a Ga ${ }^{4+}$ FIB operated at $20 \mathrm{keV}$ with a FWHM beam diameter of $619 \mathrm{~nm}$ and a beam current of $2.15 \mathrm{nA}$. There is about $15 \%$ maximum deviation between the requested (designed) and the resulting milled profiles. The authors reported that the main reasons for this difference are due to the redeposition and the instability or drift of the beam current. However, no quantitative measurement of redeposition rate or beam current instability is given. Fu et al. $[17,18]$ used a $50 \mathrm{keV} \mathrm{Ga}^{4+}$ FIB to mill a $3 \times 3$ diffractive optical element (DOE) array in a Schott BK7 glass substrate. The milling is performed using a pixel spacing of $13.7 \mathrm{~nm}$ with a beam diameter of $215 \mathrm{~nm}$ and a current of 8.66 $\mathrm{nA}$, and each element or lens has an outermost diameter of $8 \mu \mathrm{m}$ and a single DOE of $500 \mathrm{~nm}$ with a focal length of $4.8 \mu \mathrm{m}$ and a designed wavelength of $635 \mathrm{~nm}$. They also reported that the surface and dimensional quality of the optical elements is dependent on the beam size; the smaller the beam size, the better the quality $[11,18]$. Vasile et al. [19] focused on the relationship between the milling depth and the pixel dwell times which is controlled by computation program, and analyzed the formation mechanism of the profile error in the depth direction. However, the quantitative data about the profile errors and the further error compensation method were not given.

The recent generation of FIB instruments allows importing a bitmap file as a pattern to machine microstructures. The bitmap method has become increasingly popular and is currently the most commonly used processing method in FIB milling due to the convenience of usage $[12,13$, 20-22]. In the bitmap files, the milling area, beam scan routes, beam overlap ratio, and dwell time can be defined after the ion beam voltages and currents are determined. The designed profile information of 3D microstructures can be expressed by the grayscale value of each pixel in the bitmap. Fang et al. [20] used FIB bitmap patterning method to successfully fabricate nano-photomasks with $32 \mathrm{~nm}$ line width by carefully controlling the parameters such as beam dwell time, beam astigmatism, beam overlap. Chiu et al. [23] have used FIB bitmap milling to fabricate micro-lenses in silicon nitride films, and they found that the minimum resolvable zone profile is about $1 \mu \mathrm{m}$ wide and the measured numerical aperture (NA) of a $20-\mu \mathrm{m}$ micro-lens is 0.64 , closed to the design specification of 0.65 . However, the focused spot size of $0.64 \mu \mathrm{m}$ is larger than the diffraction limit of $0.49 \mu \mathrm{m}$ due to the fabrication errors in the outer rings. Sun et al. [24] proposed a divergence compensation approach based on the overlap effects and the angular-dependent sputter yield. However, the effect of redeposition was not considered in the simulation model and the sputtering rate was only simulated without verifying its effectiveness.

It is worth pointing out that there is a profile error in FIB bitmap milling of 3D microstructures due to the factors such as spatial distribution of ion beam spot, the sputter yield (depending on the incident angle), and the redeposition effect [15, 25-28]. Furthermore, the FIB milling parameters such as ion beam currents, dwell times, and beam overlap ratios also have a significant influence on the deviation between the designed and the resulting milled profiles. However, to date, few studies have been done about the machining error and its formation mechanism in FIB bitmap patterning milling, which is a necessary work to improve the fabrication accuracy of 3D microstructures. In this paper, hence, the influence of FIB milling factors and parameters on the milled profiles was investigated based on a simulation model and experimental measurements. Besides, the error formation mechanism and the error compensation strategies were also studied.

\section{Experiments and simulation methods}

\subsection{FIB bitmap milling experiments}

All of the bitmap milling experiments were carried out using a dual-beam FIB/SEM equipment (FEI Helios NanoLab G3 CX) using the liquid metal $G a$ as the ion source. The samples used in the experiment were intrinsic silicon. The ion beam acceleration voltage is set as a constant value of $30 \mathrm{kV}$ in all experiments, and the ion beam 
current is ranged from $1.1 \mathrm{pA}$ to $65 \mathrm{nA}$. The theoretical minimum beam diameter (FWHM) is $7 \mathrm{~nm}$ when the ion beam current is very low, but the actual beam diameter is varied with the ion beam current. The FIB system supports the maximum bitmap pixel of $6144 \times 4096$ and a continuous adjustable dwell time from 0.025 to $25,000 \mu$ s. In FIB milling, a one-round processing or a multi-rounds processing of scanning can be selected to reach the predetermined depth. Nevertheless, the multi-rounds processing is generally preferred with a serpentine scanning path in accurate FIB fabrication due to the slighter redeposition effect. The profiles of the 3D microstructures are measured by a white light interferometer (WLI, ZYGO NewView 9000 ). This equipment's vertical resolution and lateral resolution are $0.008 \mathrm{~nm}$ and $0.34 \mu \mathrm{m}$, respectively.

\subsection{Simulation methods}

The prediction or simulation model of the profile machined by FIB bitmap milling was established based on the method proposed by Kim et al. [29, 30]. The simulation was operated in Python. Figure 1 shows the simulation procedure of the profiles machined by FIB bitmap milling. The model initialization was firstly conducted, including importing the third-party packages and defining some known parameters. Then, the bitmap file was selected and loaded, in which bitmap pixels, ion beam currents, dwell times, and processing rounds can be defined. The bitmap pixels and dwell times can be transformed to dwell time matrices, and the size of ion beam spots depends on the ion beam currents. Finally, the multi-rounds processing was operated. During each single round, the point represented by the dwell time matrices was scanned by ion beam in the way of point by point, and then, calculated incident angles of ion beam, a calculated sputter yield, and a redeposition effect were considered and superimposed on the designed profiles. The detailed simulation method is described in Refs. [25, 30].

\section{Results and discussion}

\subsection{Influence of FIB milling parameters on the profiles}

A FIB bitmap milling experiment of a concave spherical micro-lens was carried out to clarify the influence of ion beam currents, dwell times, and beam overlap ratios on the surface quality and the shape accuracy. Figure 2 shows the designed concave spherical micro-lens. The radius, the spherical cap height, and the lateral feature size are $30 \mu \mathrm{m}$, $4.02 \mu \mathrm{m}$, and $30 \mu \mathrm{m}$, respectively.

\subsubsection{Dwell times}

The ion beam remains in each pixel for a particular time before it jumps to the next pixel. This time is defined as the beam dwell time. In the bitmap file, the grayscale value $(0-255)$ of each pixel determines the dwell time. If the grayscale value is set to be 0 (black), the corresponding dwell time is 0 , which means the material will not be removed; if the grayscale value is set to be 255 (white), the corresponding user-defined maximum dwell time is used. As a result, the ion beam at the white position has longer dwell time than the one at the black position in FIB bitmap milling. The dwell time corresponding to the grayscale value is linearly interpolated on the basis of the value between 0 and the maximum dwell time. In these tests, the maximum dwell times $1 \mu \mathrm{s}, 10 \mu \mathrm{s}$, and $100 \mu \mathrm{s}$ were used to mill micro-lenses and the ion beam current and the beam spot overlap ratio are set as $21 \mathrm{nA}$ and $50 \%$, respectively.

Figure 3 shows the SEM images and the measured profiles of the micro-lens processed by the different maximum dwell times. It can be observed that the bottom of the micro-lens is a smooth spherical shape when the maximum dwell time is $1 \mu \mathrm{s}$. As the maximum dwell time increased to $10 \mu \mathrm{s}$, a little of residue appeared at the bottom, which can be resulted from the redeposition of the sputtered materials [23]. When the maximum dwell time increased to $100 \mu \mathrm{s}$, the volume of the residue increased at the bottom. It can be seen that the redeposition effect can be alleviated by using a shorter dwell time, which gave rise to an excellent surface quality. However, the time resolution of bitmap is insufficient when the dwell time is too short, which leads to an obvious step mark on the profile as shown in Fig. 3. Furthermore, a shorter dwell time caused a lower milling efficiency since the number of the processing round increases with the decreasing dwell time in the multi-rounds FIB milling for a designed machining depth.

\subsubsection{Ion beam currents}

As the ion beam current increases, both the number of ions incident per unit time and the ion beam diameter increase. Therefore, three ion currents, $9.3 \mathrm{nA}, 21 \mathrm{nA}$, and $47 \mathrm{nA}$ were selected to investigate the influence of ion beam currents on FIB milling. The theoretical ion beam diameters corresponding to these three currents are $249 \mathrm{~nm}, 400 \mathrm{~nm}$, and $820 \mathrm{~nm}$, respectively. The other two parameters, the dwell time and the beam overlap ratio are set as $10 \mu$ s and $50 \%$, respectively. Since the milling area of the bitmap file is set as $32 \mu \mathrm{m} \times 32 \mu \mathrm{m}$, the pixel sizes of the bitmap corresponding to the three currents are $267 \times 267,160 \times 160$, and $80 \times 80$, respectively. That is, 


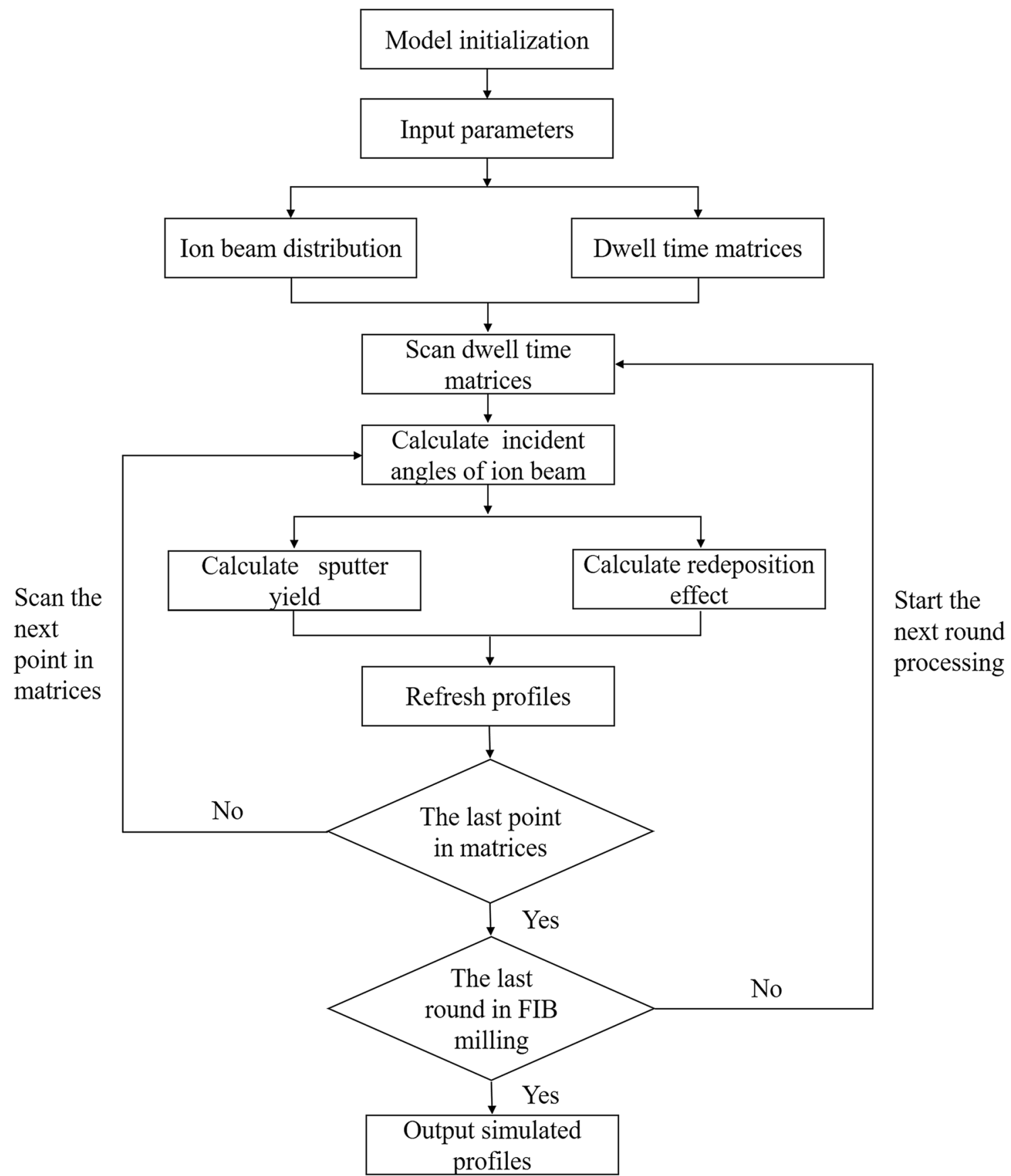

Fig. 1 Flowchart of the profiles simulation in FIB bitmap milling

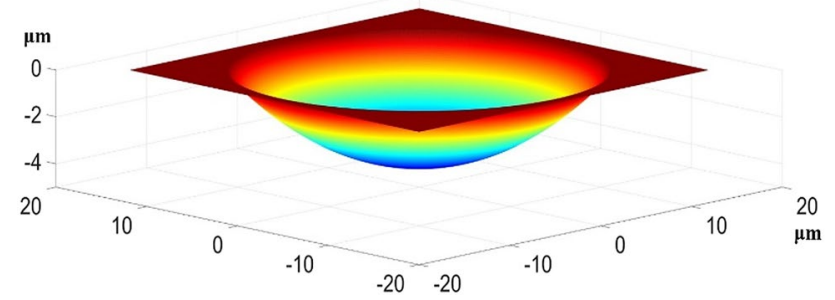

Fig. 2 The schematic of the designed concave spherical micro-lens

SN Applied Sciences the pixel density gradually decreased as the ion beam current increased, as shown in Fig. 4. The three bitmaps were loaded in FIB system to carry out the milling process. The processing time for each ion beam current was $600 \mathrm{~s}, 273 \mathrm{~s}$, and $186 \mathrm{~s}$, respectively. Figure 5 shows the SEM images and the measured profiles of the micro-lens processed by different ion beam currents. It can be observed that the edge curvature of the concave micro-lens gradually raises with the increase in the ion beam current, which meant 

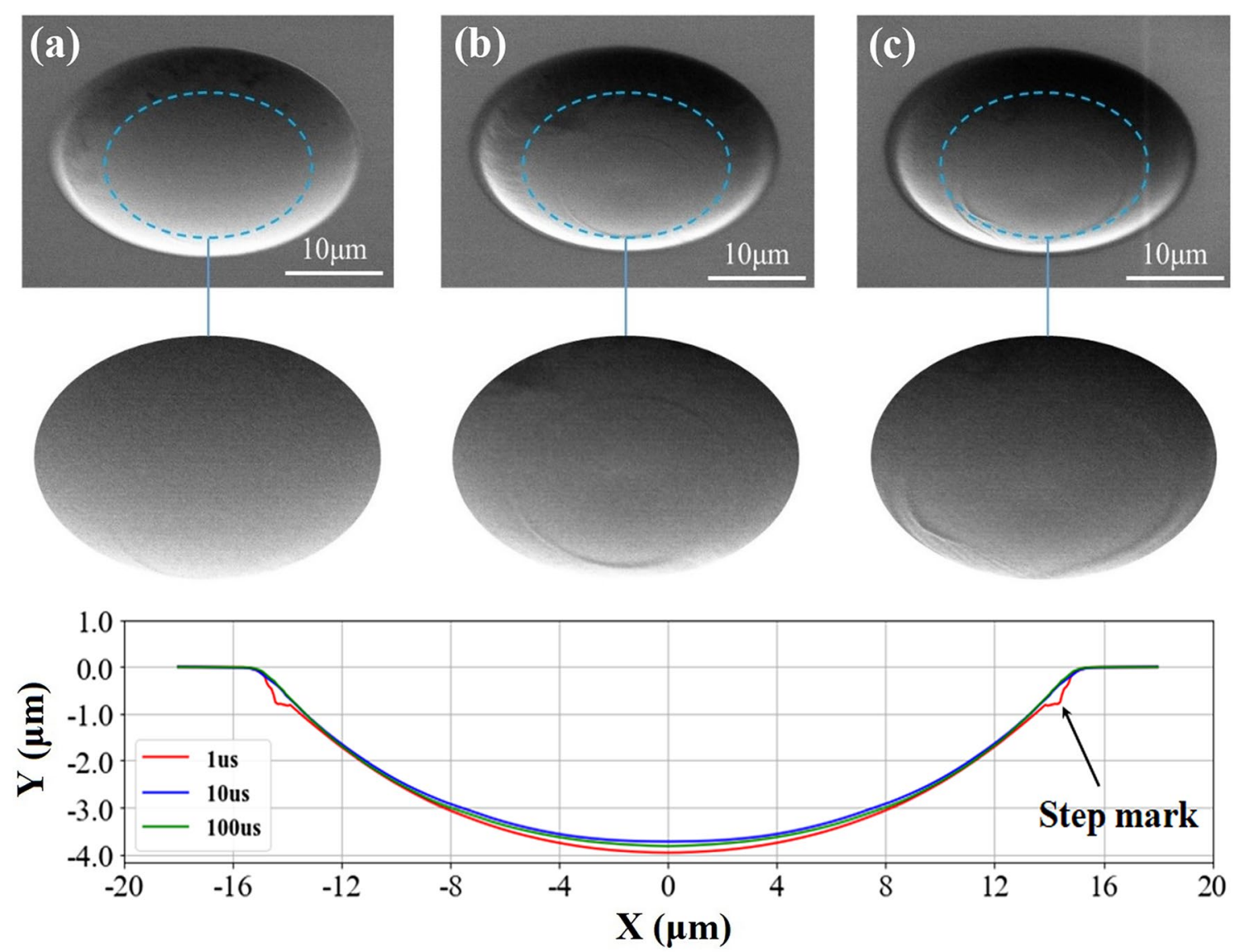

Fig. 3 The micrographs and the measured profiles of the micro-lens processed by the different maximum dwell time: a $1 \mu \mathrm{s} ; \mathbf{b} 10 \mu \mathrm{s} ;$ and $\mathbf{c}$ $100 \mu \mathrm{s}$

Fig. 4 The bitmaps with different pixel sizes used for FIB milling micro-lens
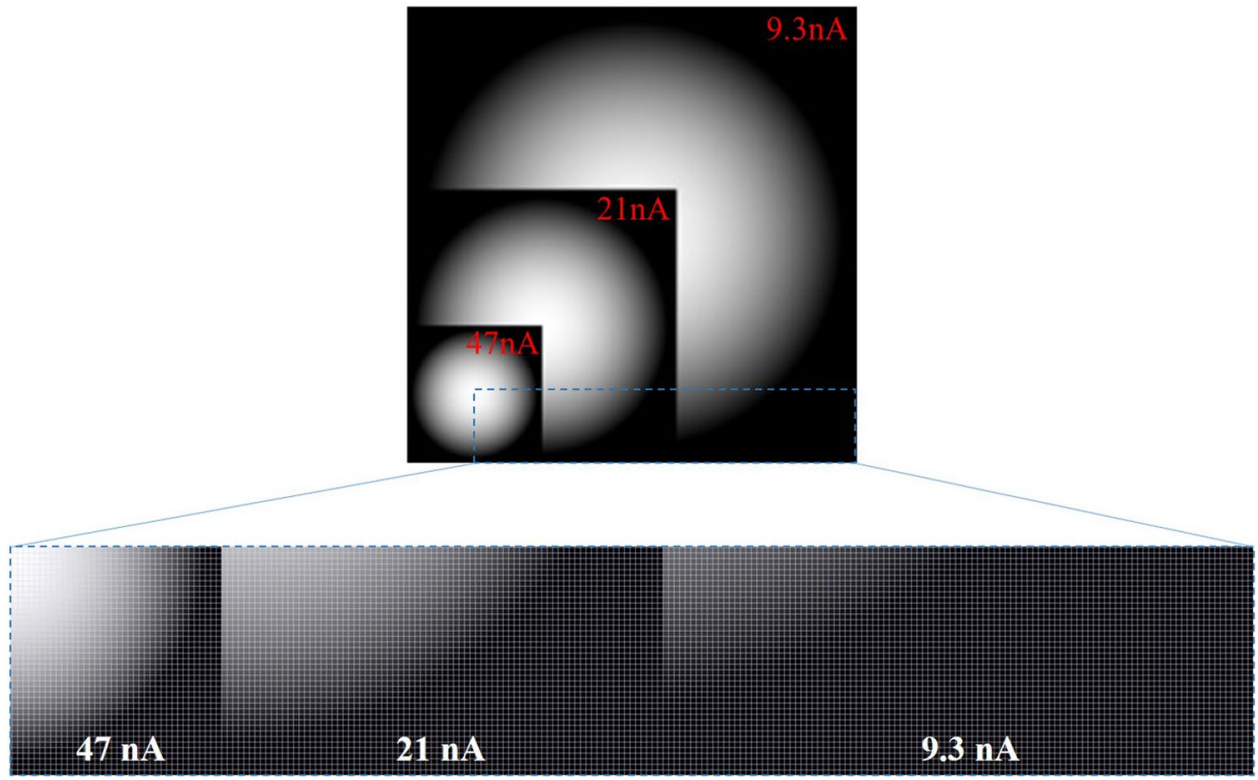


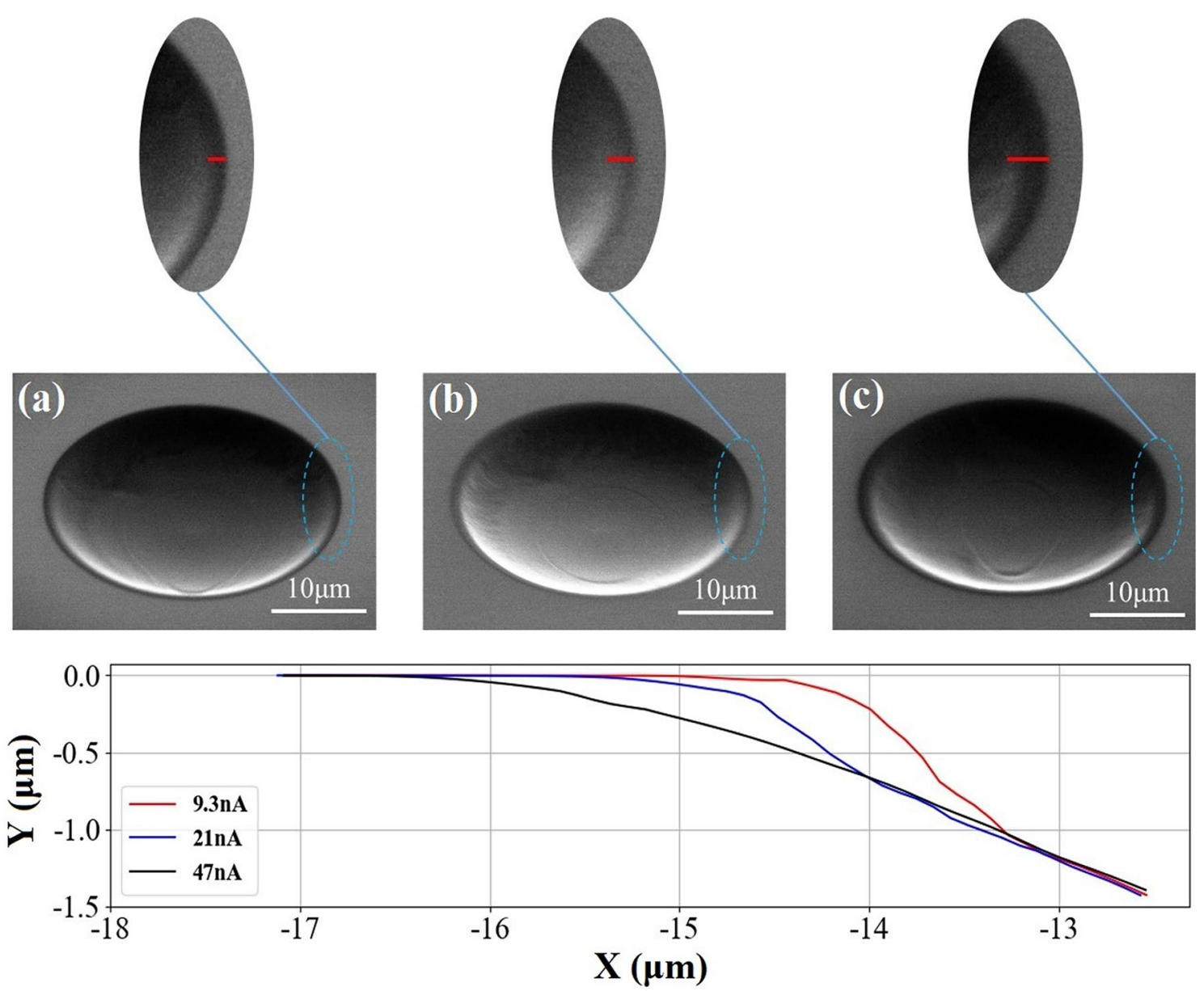

Fig. 5 The micrographs and edge profiles of the micro-lens milled by different ion beam currents: a $9.3 \mathrm{nA} ; \mathbf{b} 21 \mathrm{nA}$; and c $47 \mathrm{nA}$

that the profile error gets larger. The decline of the milling accuracy can be attributed to the increase in ion beam current leading to a larger ion beam diameter although it also improves the FIB milling efficiency.

\subsubsection{Beam overlap ratios}

The beam overlap ratio is the ratio of the ion beam moving distance to the ion beam diameter. In FIB bitmap milling, the move distance of the ion beam for each step depends on the bitmap pixels density and the ion beam diameter which are related to the beam current. Thus, the ion beam overlap ratio is proportional to the bitmap pixels density when the ion beam current is constant. In these tests, the ion beam current and the maximum dwell time are set as $21 \mathrm{nA}$ and $10 \mu \mathrm{s}$, respectively, and the theoretical ion beam diameter was $400 \mathrm{~nm}$. Three bitmaps with different pixels densities were used to mill micro-lenses. The pixels densities were set as $100 \mathrm{~nm}, 200 \mathrm{~nm}$, and $400 \mathrm{~nm}$, respectively, and the beam overlap ration can be calculated to be $25 \%, 50 \%$, and $100 \%$, respectively. As the size of the bitmap file was $32 \mu \mathrm{m} \times 32 \mu \mathrm{m}$ (milling area), the pixel sizes of the three bitmaps were $320 \times 320,160 \times 160$, and $80 \times 80$, respectively.

Figure 6 shows the SEM images of the micro-lens processed by different beam overlap ratios. It can be observed that as the beam overlap ratio increased, the ion beam move marks (just like the "feed marks" in cutting process) became visible from the front view as shown in Fig. 7a. Figure $7 \mathrm{~b}$ explains the formation mechanism of the "feed marks."When the beam diameter $(d)$ keeps constant under a certain current, the smaller beam overlap ratio $(s / d)$ is adopted, the smaller beam jumping distance between the two neighboring pixels $(s)$ is. This results in a large overlapped milled area and generated a super-smooth surface. In contrast, the larger beam overlap ratio $(s / d)$ is adopted, the larger the beam jumping distance $(s)$ is. Thus, a part of the area between the two neighboring pixels cannot be milled due to a Gaussian distribution of the ion beam energy [15], which generates the "feed marks." However, a too small beam overlap ratio gives rise to a too large 

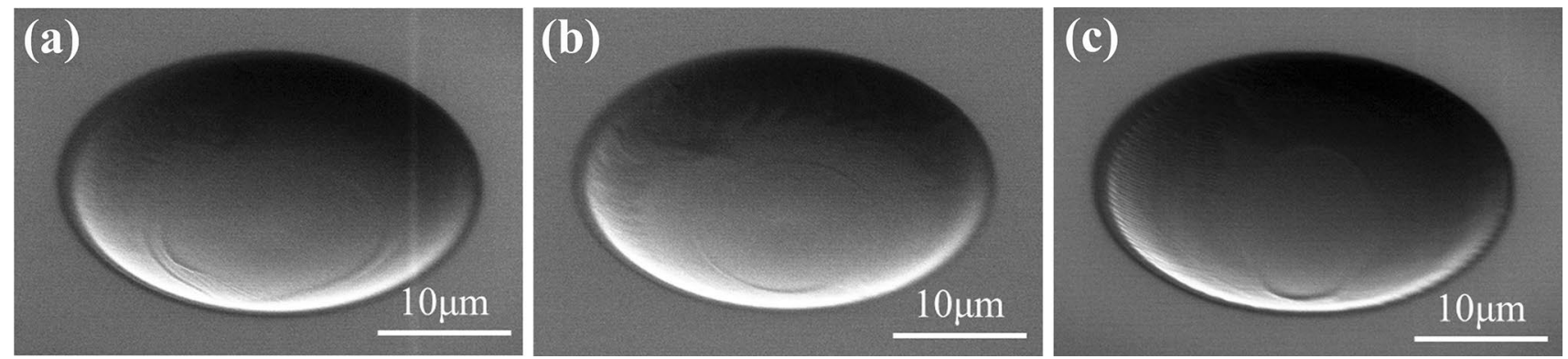

Fig. 6 The micrographs the micro-lens processed by different beam overlap ratios: a 25\%; b 50\%; and c 100\%

Fig. 7 a The front view of the micro-lens processed with $100 \%$ beam overlap ratio and $\mathbf{b}$ the schematic of beam overlap ratios
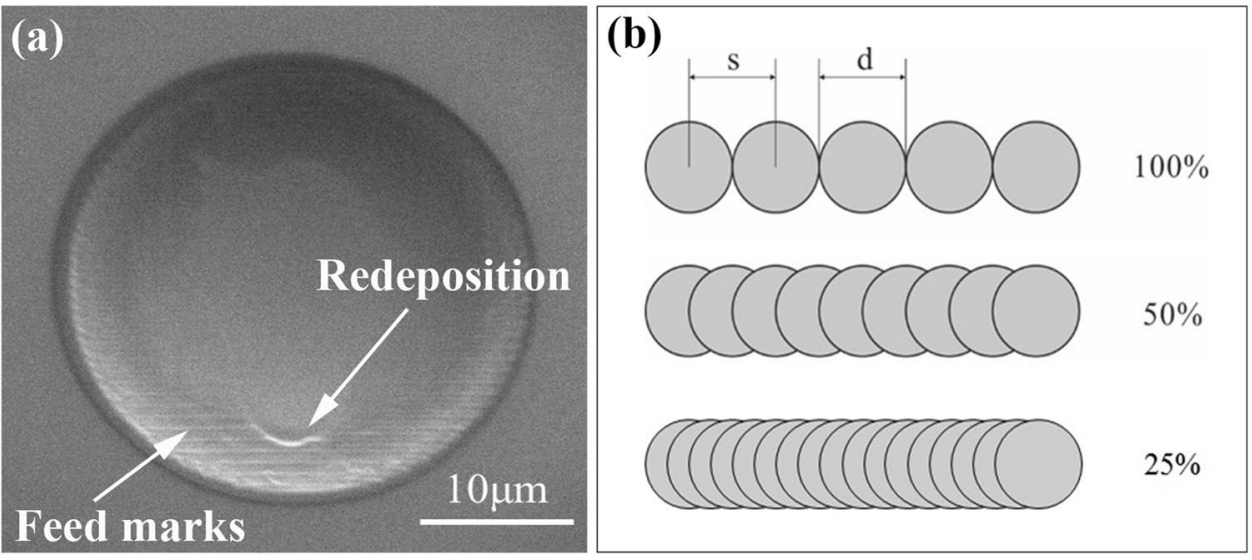

bitmap file which cannot be read by the FIB system and declines the processing efficiency.

\subsection{Surface profile simulation and formation mechanism of profile error}

In order to investigate the prediction accuracy of the simulation model, a comparison analysis between the measured (experimental) profiles and the simulated profiles is carried out. The concave spherical micro-lens in Fig. 2 is processed by FIB bitmap milling with the following parameters: Ion beam voltage is $30 \mathrm{kV}$; ion beam current is $21 \mathrm{nA}$; the bitmap size is $32 \mu \mathrm{m} \times 32 \mu \mathrm{m}$; the maximum dwell time is $10 \mu \mathrm{s}$; the beam overlap ratio is $50 \%$; and the processing rounds are 1000,2000 , and 3000, respectively.

Figure 8 a shows the measured and simulated profiles of the micro-lens milled with different processing rounds. The results indicated that the simulated profiles agree well with the measured profiles. However, it is worth noting that a flat bottom can be observed visibly from the profile curves when the number of the processing round exceeded 2000. This phenomenon is attributed to the redeposition effect that will be studied in the following. Figure $8 \mathrm{~b}$ gives the error curves of the measured profile and the simulated profile, which exhibits the deviations of the two kinds of profiles from the designed (ideal) profile. The maximum positive deviation between the measured profile and the designed profile was located at the edge of the concave micro-lens, and the value was $0.21 \mu \mathrm{m}$ while the maximum negative deviation was located at the bottom of the concave micro-lens and the value was $0.23 \mu \mathrm{m}$. From the profile data, the shape accuracy $P-V$ value for the 3000 rounds of processing was calculated to be $0.44 \mu \mathrm{m}$ and the RMS value was calculated to be $0.142 \mu \mathrm{m}$. It also can be observed in Fig. 8b that the two error curves were also very similar (the calculated $P-V$ value and RMS value for the simulated profile were $0.37 \mu \mathrm{m}$ and $0.122 \mu \mathrm{m}$, respectively), which indicated a high accuracy of the simulation model.

From the FIB bitmap milling experiment results, it can be found that the ion beam diameter and the redeposition effect had a great influence on the profile error. Besides, the sputter yield, defined as the number of atoms ejected from the target surface per incident ion, changes with the incident angle of the ion beam in processing of $3 \mathrm{D}$ microstructures, and it also affects the processed profiles significantly in FIB milling. Figure 9 shows a schematic for elucidating the effect of the three factors on the FIB milling processing. However, these factors are difficult to adjust and control by means of experiments. Thus, in order to 

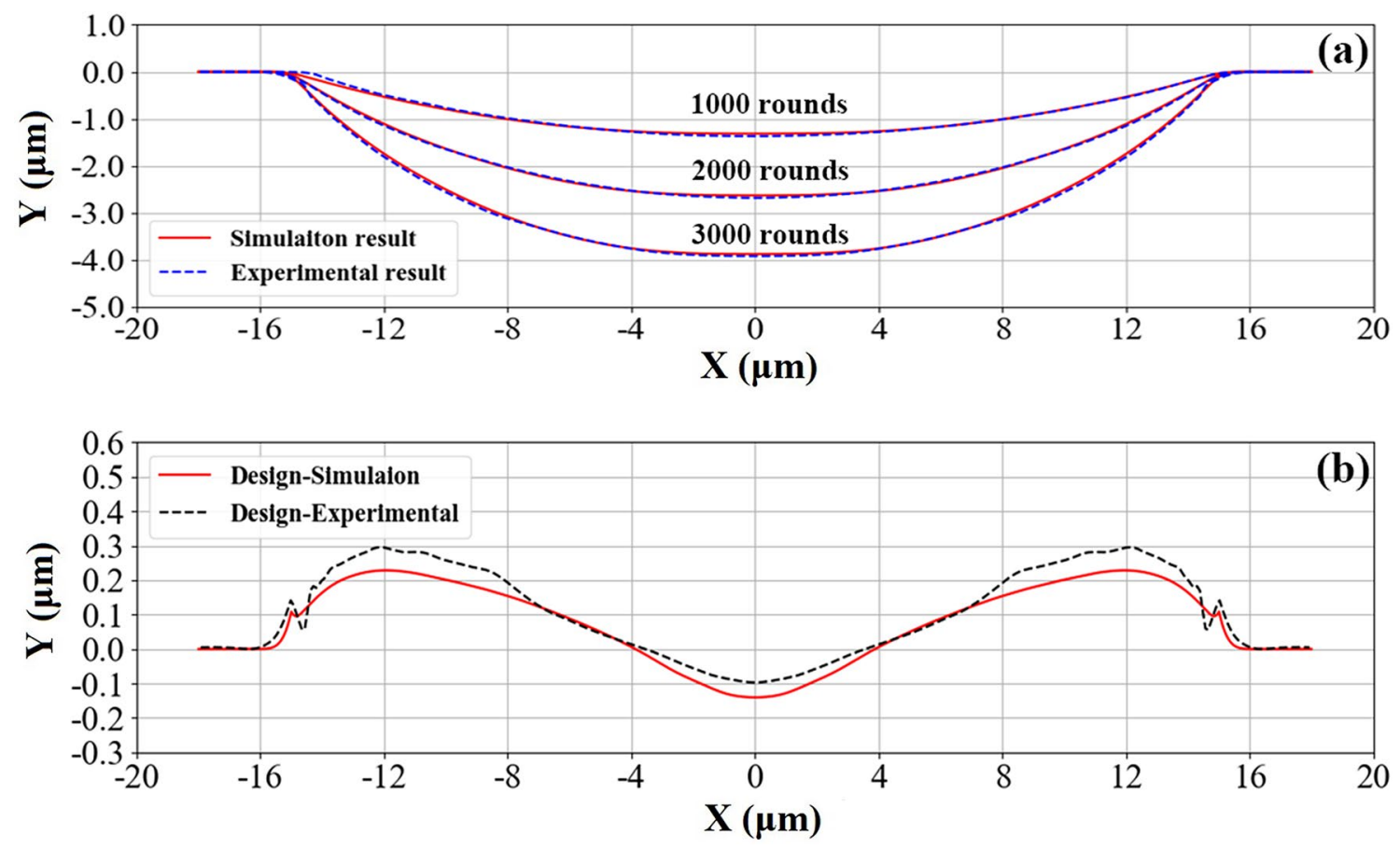

Fig. 8 The simulated and measured profiles and their error curves: a the comparison between the simulated and the measured profiles processed with different processing rounds and $\mathbf{b}$ the error

clarify the role of these implicit factors in FIB milling, the simulation method is used to study the effect of the three factors on the milled profiles.

\subsubsection{Redeposition effect}

Figure 10a shows the comparison between the ideal profile and the simulated profile without the consideration of redeposition. It can be observed that the flat bottom disappeared when the redeposition effect was removed and the two profile curves agree well. From the error curves in Fig. 10b, the negative deviation decreases significantly without the redeposition effect. The accuracy of the simulated profile is $0.25 \mu \mathrm{m}$ of $P-V$ value and $0.108 \mu \mathrm{m}$ of RMS value. Compared to the original simulated profile with the redeposition effect, the two values decreased by $32 \%$ and $11 \%$, respectively.

\subsubsection{Sputter yield}

The relationship between the sputter yield $(Y)$ and the ion incident angle $(\theta)$ can be expressed by the following equation [31].

$Y(\theta)=Y(0) \cos (\theta)^{-f} e^{\left(-c\left(\cos (\theta)^{-1}-1\right)\right)}$ curves comparison between the simulated and the measured profile. The error curve gives the deviation between the simulated/ measured profile and the ideal profile

$Y(0)$ is the sputter yield when the ion incident angle is zero. By setting the incident angle to zero, then $Y(\theta)=Y(0)$, which is a constant value. Thus, the influence of sputter yield on the profile error can be studied by comparing the simulated profiles with a constant sputter yield and a variable sputter yield. Moreover, the effect of redeposition was also removed. Figure 11a shows the comparison between the designed profile and the simulated profile with a constant sputter yield, and it can be observed that these two profiles are very similar. Figure $11 \mathrm{~b}$ shows the error curve of the simulated profile approaches to the zero line and the $P-V$ and RMS value are $0.10 \mu \mathrm{m}$ and $0.056 \mu \mathrm{m}$, respectively. Compared to the original simulated profile, these two values decreased by $77 \%$ and $61 \%$, respectively. Nevertheless, the profile error still remained, which may be attributed to the spatial distribution of ion beam in FIB bitmap milling and the limited accuracy of the simulation model.

\subsubsection{Ion beam diameter}

The wastage of FIB system components such as the optical gate leads to the widening of the beam spot, which means the distribution of ion density becomes sparser. In view of this, the ion beam diameters (FWHM) $800 \mathrm{~nm}$, $1200 \mathrm{~nm}, 1600 \mathrm{~nm}$, and $2000 \mathrm{~nm}$ were selected to clarify the effect of widened ion beam on the processing profile 
(a) Incident ion beam

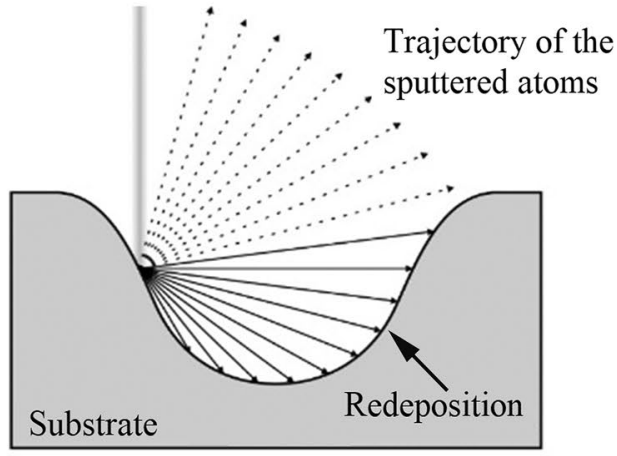

(b)

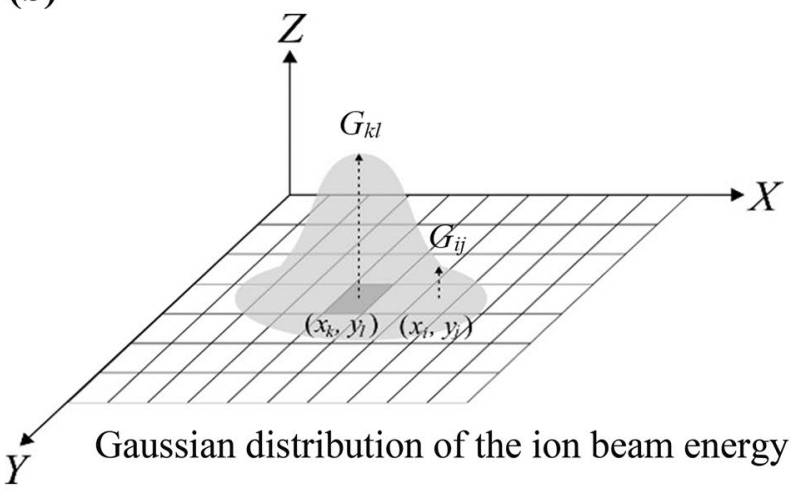

(c)

Incident ion beam

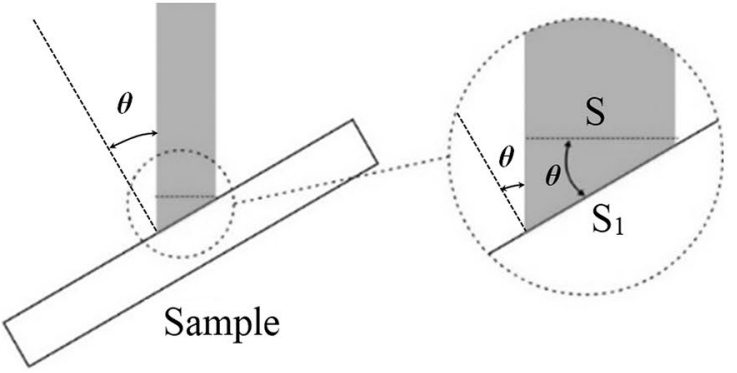

Fig. 9 The schematic for elucidating the effect of the three factors on the FIB milling processing: $\mathbf{a}$ the generation of the redeposition; $\mathbf{b}$ the distribution of ion beam energy; and $\mathbf{c}$ the sputter yield depending on the incident angle of ion beam
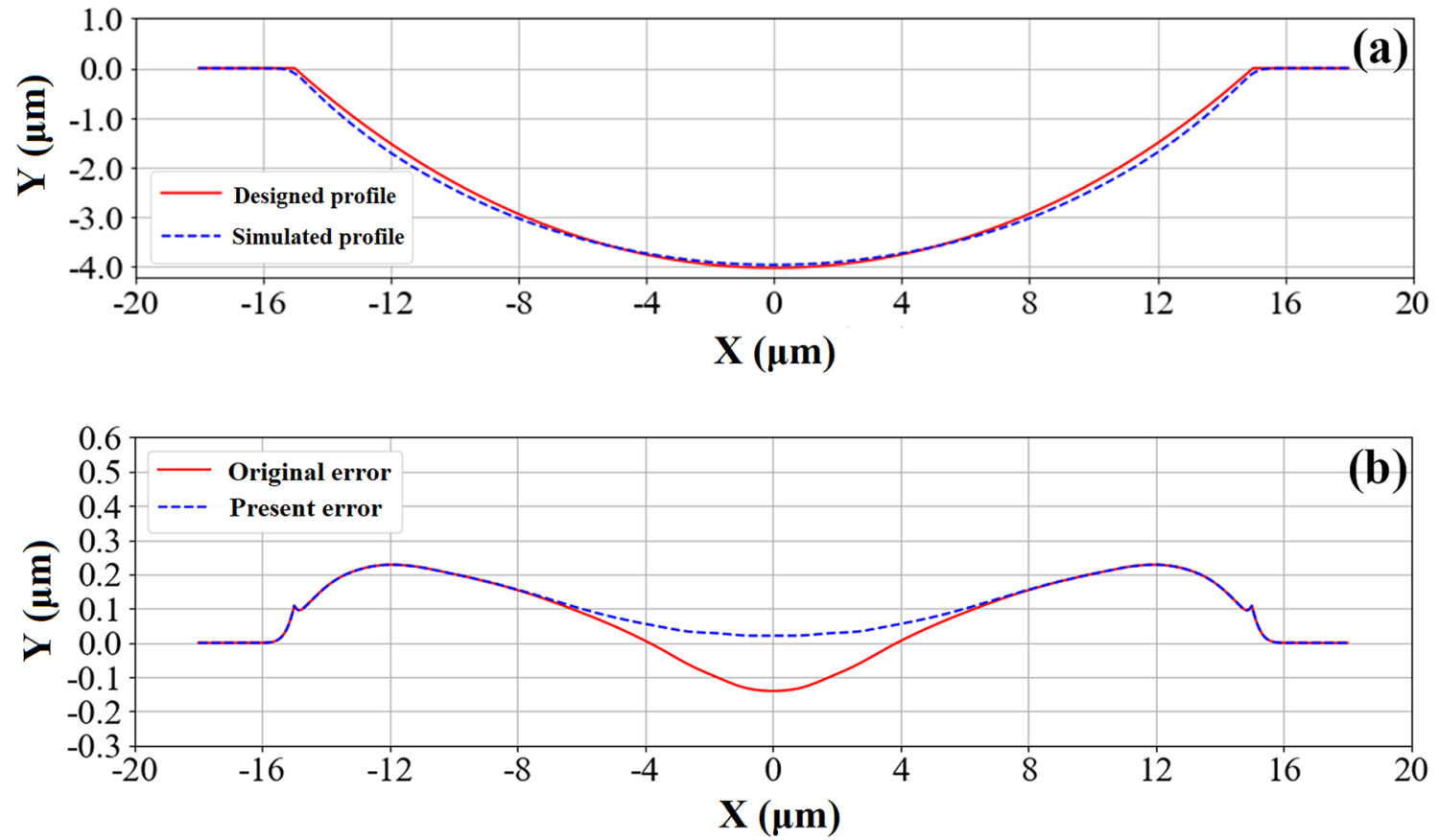

Fig. 10 The simulated profile and its error curve without the redeposition effect: a the comparison between the simulated and the design profile; $\mathbf{b}$ the comparison between the profile error curves before and after removing redeposition effect 

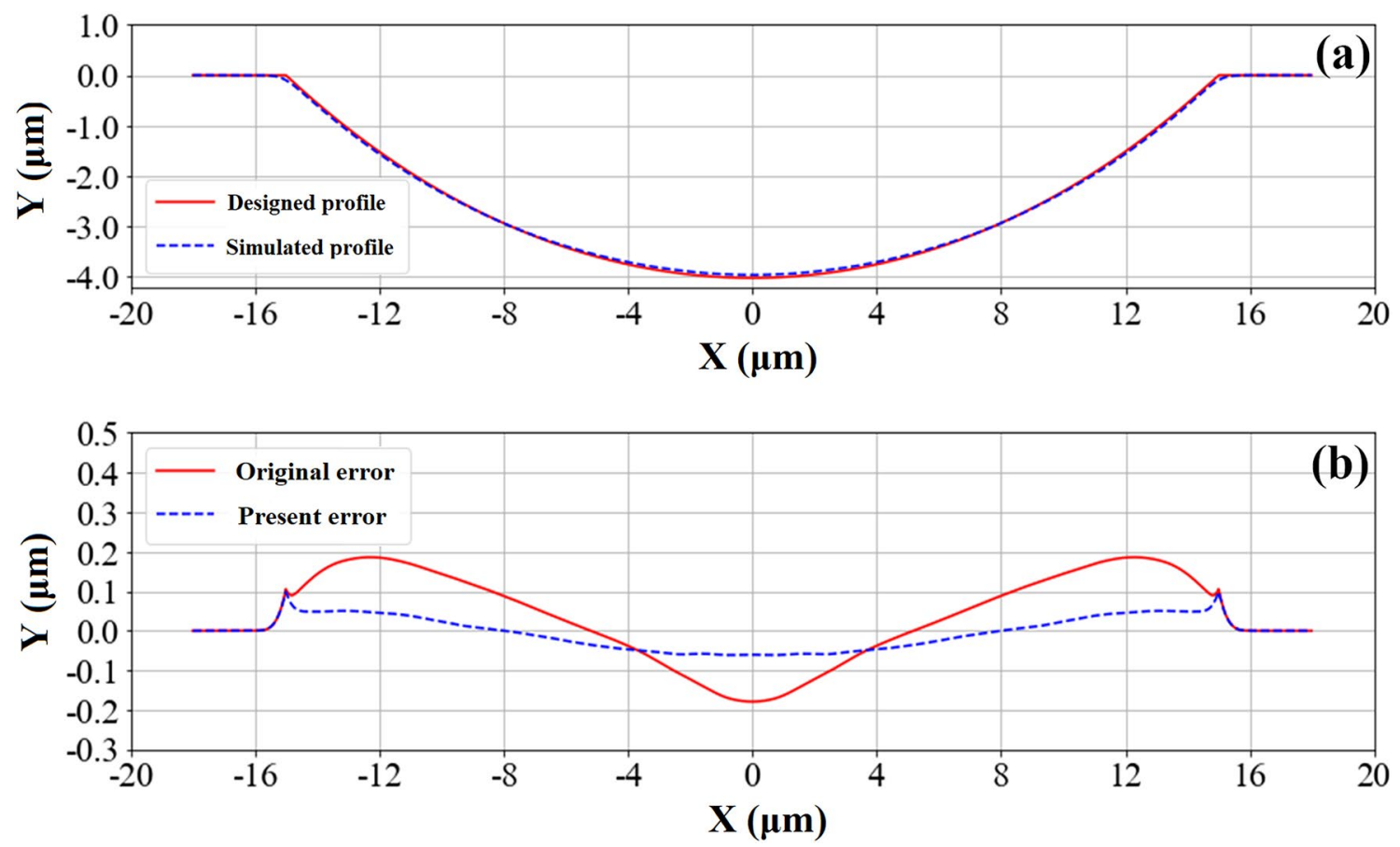

Fig. 11 The simulated profile and its error curve without the effects of sputter yield and redeposition: a the comparison between the simulated and the design profile; $\mathbf{b}$ the comparison between the

profile error curves before and after removing the effect of sputter yield and redeposition
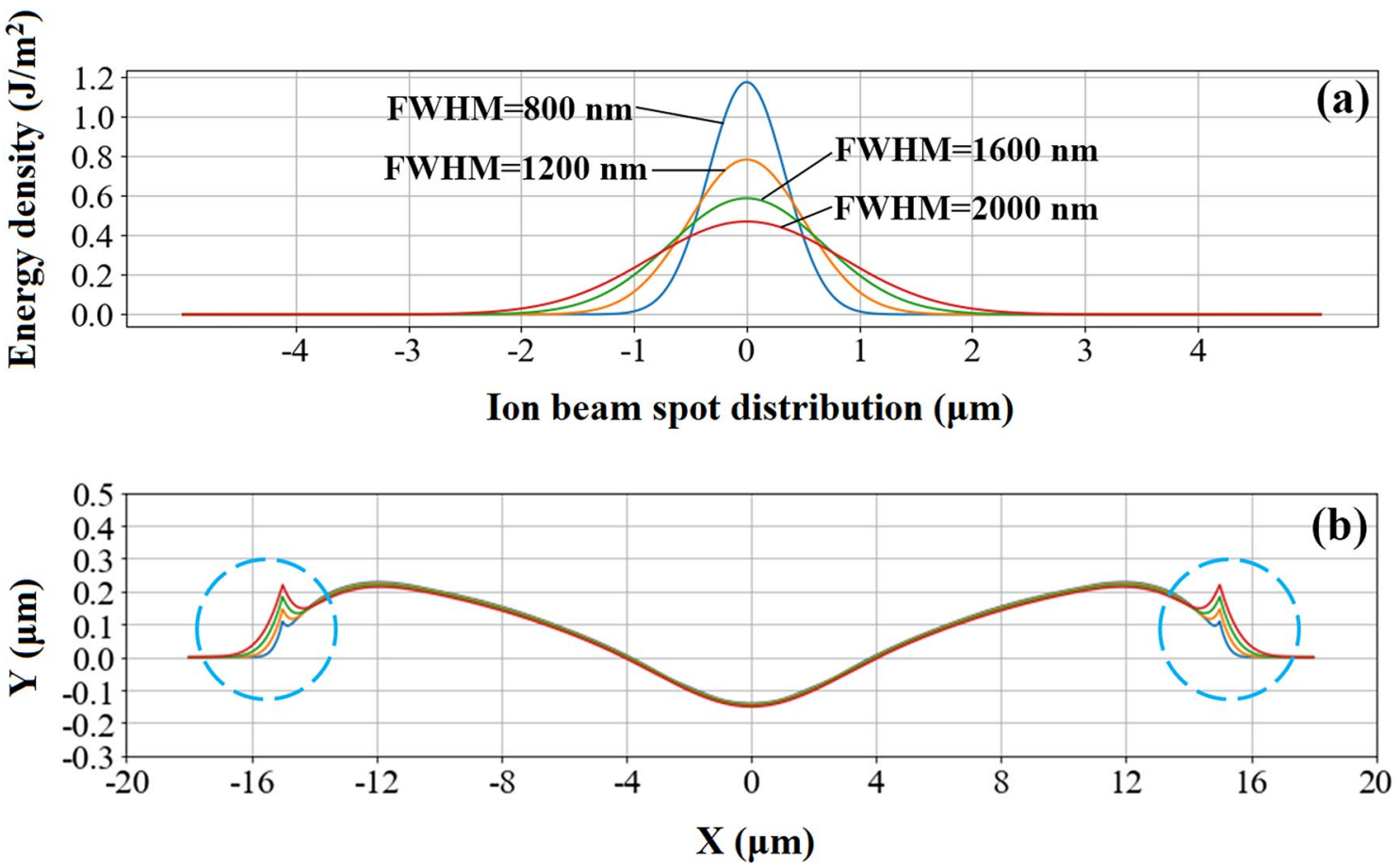

Fig. 12 a The Gaussian distribution of the ion beam energy and $\mathbf{b}$ the error curve of the profiles simulated with different ion beam diameters 
by the simulation model. The Gaussian distributions for the ion beam with different diameters are shown in Fig. 12a, and the error curves of the simulated profiles processed with different ion beam are shown in Fig. 12b. It can be observed that the widened ion beam causes a profile error at the site of the micro-lens edge and the error value is directly proportional to the ion beam diameter. However, the error caused by widened ion beam is inevitable because of the hardware degeneration.

\subsection{Error compensation}

In the process of profile simulation of FIB bitmap milling, the function that describes the sputter yield and the redeposition effect is complex (including exponential function, logarithmic function, trigonometric function, inverse trigonometric function, multiple derivative, and integral). Besides, the sputtering yield of the next processing rounds depends on the profile of the previous processing round. Thus, it is difficult to use mathematical analytic method to get the corrected bitmap file by inputting designed profiles bytes into the prediction simulated model and then reverse-deducing a modified bitmap. Here, two methods are proposed to modify the original bitmap to compensate the profile error.

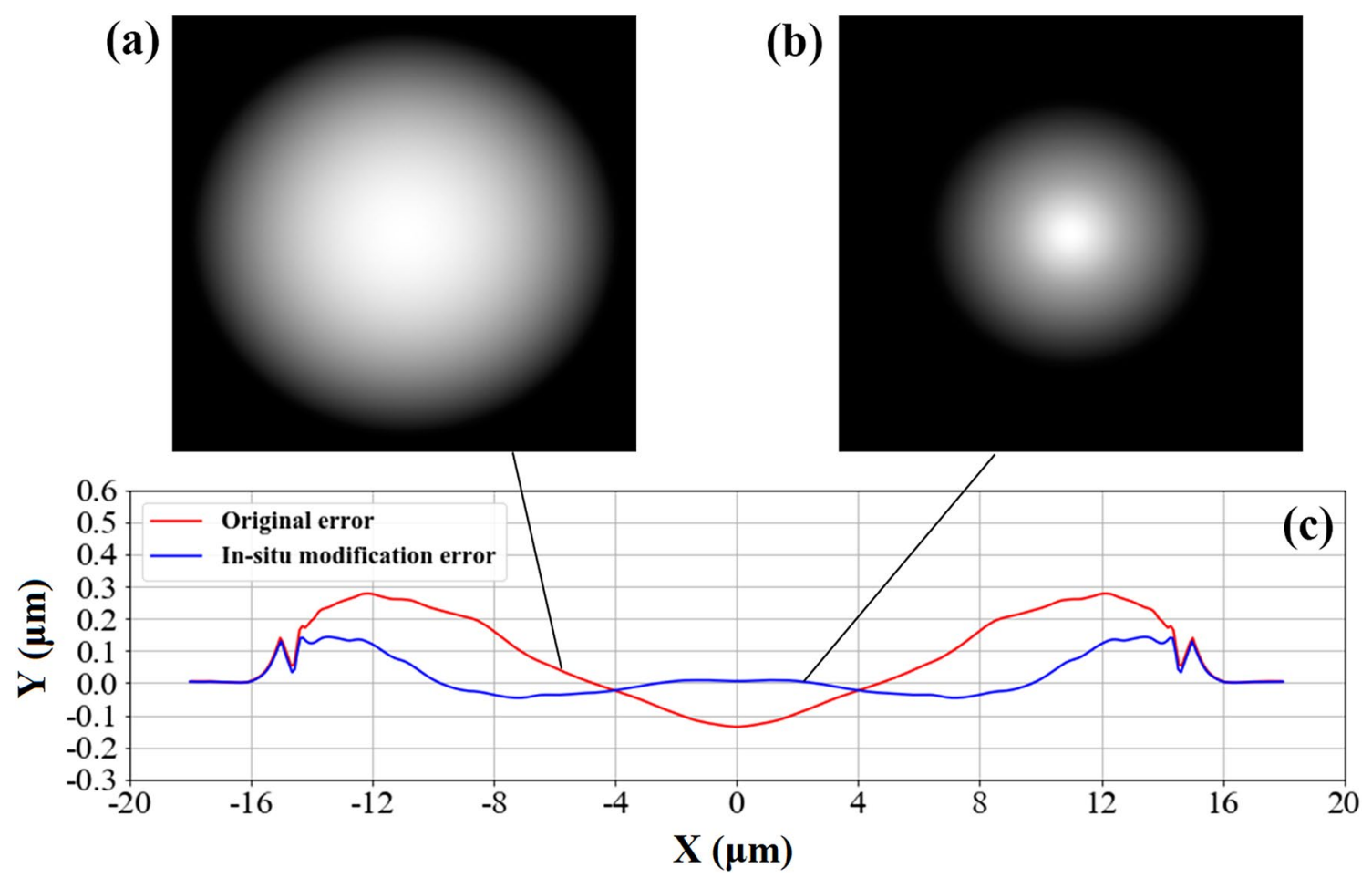

Fig. 13 The in situ modification method: $\mathbf{a}$ the original FIB milling bitmap; $\mathbf{b}$ the substituted bitmap used for in situ modification; and $\mathbf{c}$ the comparison of the error curves between the original milled profile and the compensated profile

\subsubsection{In situ modification}

FIB milling has the feasibility of superposition processing by multiple bitmap files. Thus, an in situ modification method is proposed and its basic steps are as follows: (1) simulating with an original designed bitmap; (2) stopping the simulation after $n$ rounds processing and calculating the simulated profile error; (3) the two-dimensional error curve transforms into the three-dimensional profile value through the interpolation method and then transforms into the bitmap map used for modifying profile; calculating the remaining processing rounds number (assuming that the remaining round number is $m$ ); (4) FIB milling with these two bitmap files with $n$ rounds processing and $m$ rounds processing, respectively.

The concave micro-lens is taken as an example. As shown in Fig. 8, 3000 rounds of processing are needed to achieve the design profile. In order to ensure the compensated accuracy and efficiency, the error should be as small as possible. Therefore, 2800 rounds ( $n$ rounds) are selected as the interrupted processing round. The error curves of the temporary simulated profile can be used to the modified bitmap file for the subsequent processing. Thus, this $2 \mathrm{D}$ error curve can be transformed into the 3D profile value and then into bitmap file by interpolation method as shown in Fig. 13b. This bitmap file is used for the remaining 
200 rounds of processing ( $m$ rounds). In order to verify the in situ modification compensation method, the FIB milling experiments were carried out and the profile of the microlenses was measured. From the comparison of the profile error curves before and after compensation shown in Fig. 13c, it can be found that the error deceases at almost every surface site of the micro-lens. The shape accuracy of the compensated profile is $0.19 \mu \mathrm{m}$ for $P-V$ value and $0.062 \mu \mathrm{m}$ for RMS value. These two values decreased by $57 \%$ and $56 \%$, respectively, compared to the $P-V$ value $(0.44 \mu \mathrm{m})$ and RMS value $(0.142 \mu \mathrm{m})$ before compensation. However, this method needs to replace a bitmap file in the FIB milling processing, which reduces the simplicity and convenience of the bitmap method. Furthermore, the method also can not compensate the negative profile errors as FIB milling is a kind of material reduction manufacturing technology.

\subsubsection{Iterative optimization}

Figure 14 shows the detailed steps of the iterative optimization method: (1) inputting an originally designed bitmap file into simulation model; (2) computing the profile error of the simulated profile; and (3) determining whether the shape accuracy reaches an acceptable level, i.e., less than a certain threshold value. The iteration stops if it is reached, and the current used bitmap file is the final one used for milling. If not, a modified bitmap, which is improved based on the present profile error, is inputted into the simulation model and the procedure goes back to the initial step until the iteration stops.

The milling of the concave micro-lens is also taken for example. The original bitmap is shown in Fig. 13a. Then, the bitmap is inputted into the simulation system. As shown in Fig. 8, the $P-V$ value is $0.37 \mu \mathrm{m}$ at this time. If the stated threshold of $P-V$ value is $0.2 \mu \mathrm{m}$, the bitmap modification program started and gets a new bitmap shown in Fig. 15a. The detailed modification procedures of the bitmap are as follows: (1) the ordinate of the profile error divides the height of the micro-lens and the obtained values converted into the integers between 0 and 255 , which is the corrected values of the original bitmap; (2) the original bitmap matrix minuses the corrected values and then zooms out to $0-255$ (required by grayscale image coding) to obtain the modified bitmap used for the next round of iteration. The difference between the two grayscale images is slight and not intuitive. To distinguish them, the profile error matrix of the original and the new bitmap file is extracted as shown in Fig. 15b. When the new bitmap is inputted into the simulation model, the surface accuracy of the simulation profile is $0.10 \mu \mathrm{m}$ for $P-V$ value. The value is less than the threshold value of $0.2 \mu \mathrm{m}$, and thus, the iterative optimization process is stopped. This current bitmap file is outputted as the final bitmap for FIB milling. The profile error curves before and after compensation are compared in Fig. $15 \mathrm{c}$. The shape accuracy after compensation is $0.13 \mu \mathrm{m}$ for $P-V$ value and $0.064 \mu \mathrm{m}$ for RMS value, which decreased by $71 \%$ and $54 \%$, respectively.

In order to compare the two error compensation methods, the FIB bitmap milling experiments of a sinusoidal and a concave cylindrical microstructures were also carried out. The SEM images of sinusoidal and concave cylindrical microstructures for normal processing, in situ modification, and iterative optimization are shown in Figs. 16 and 17 , respectively. The samples' profile error curves are also compared in Fig. 18, and it can be found that both of the two compensation methods have achieved good results which are listed in Table 1. It is worth noting that the in situ modification method is better than the iterative optimization method in the aspect of improving of the RMS value which indicates a global error of the profiles. But the iterative optimization method only needs one

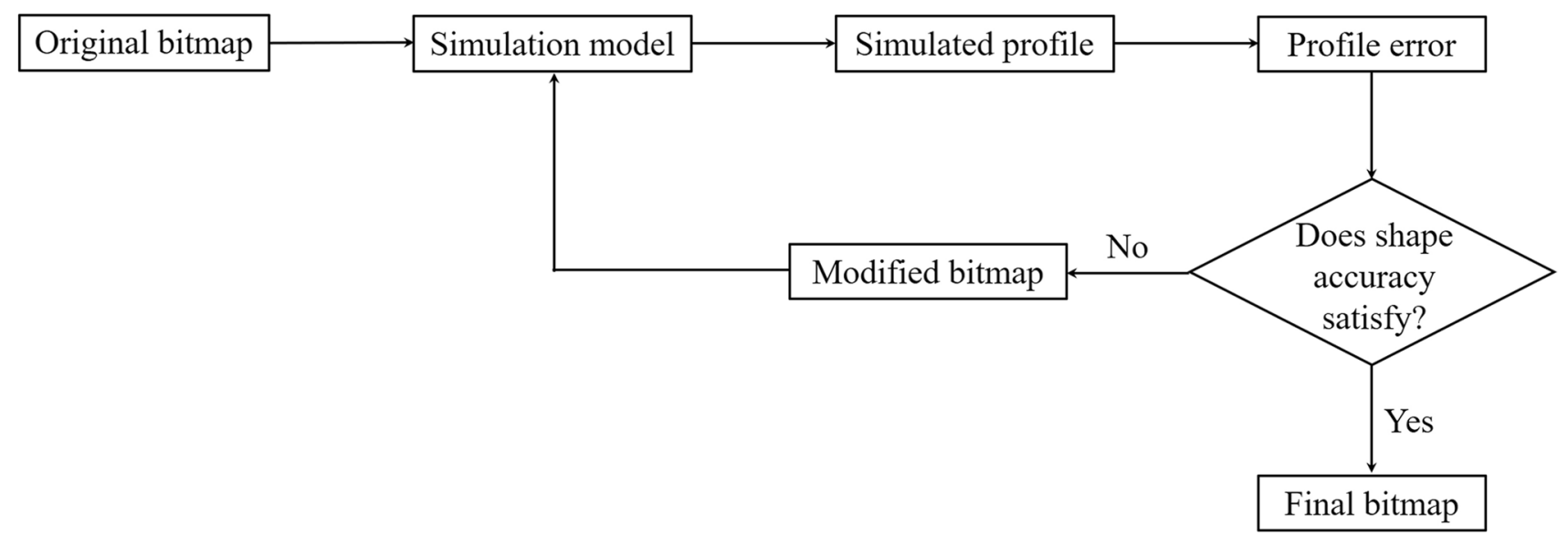

Fig. 14 Flowchart of iterative optimization method

SN Applied Sciences 
(a)

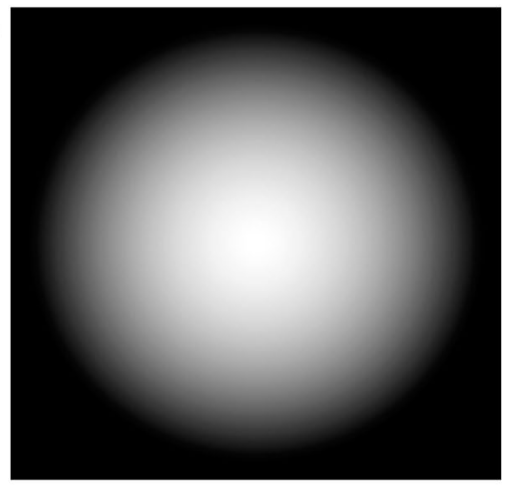

(b)

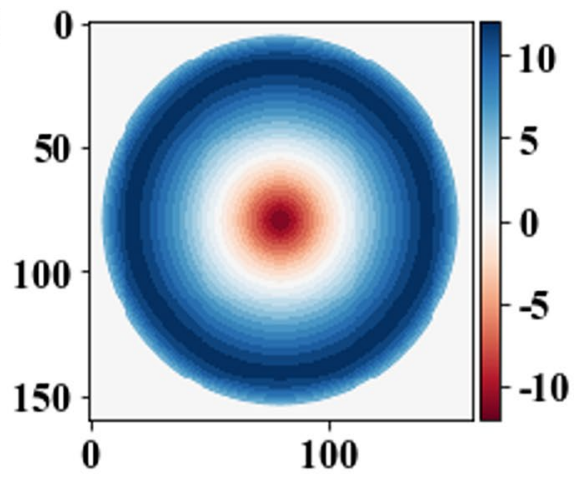

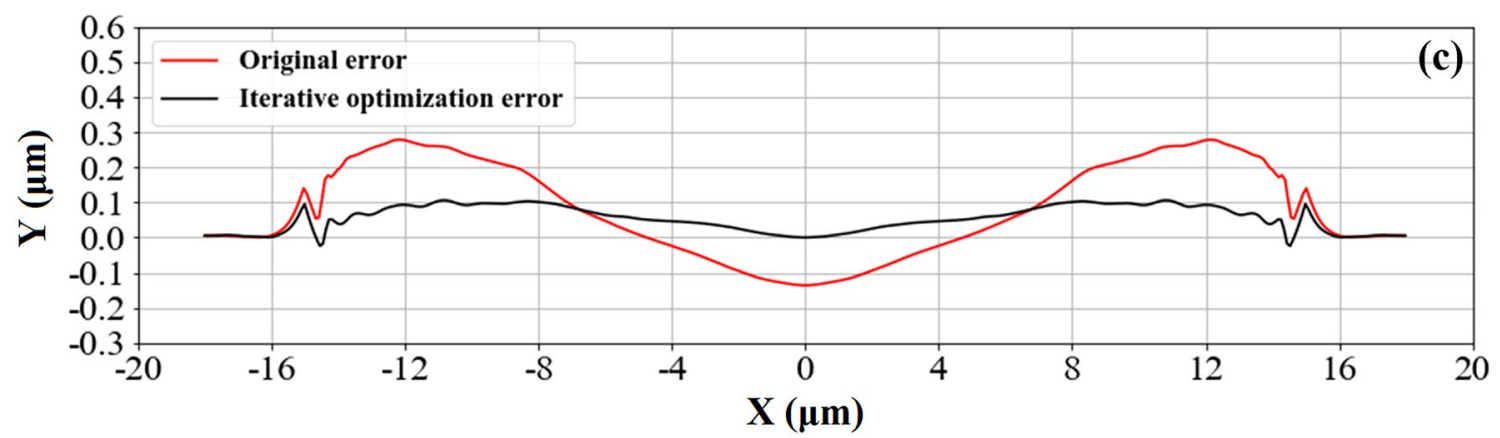

Fig. 15 The iterative optimization method: $\mathbf{a}$ the optimized bitmap used for modification; $\mathbf{b}$ the difference matrix for the original bitmap and the optimized bitmap; and $\mathbf{c}$ the comparison of the error curves between the original milled profile and the compensated profile
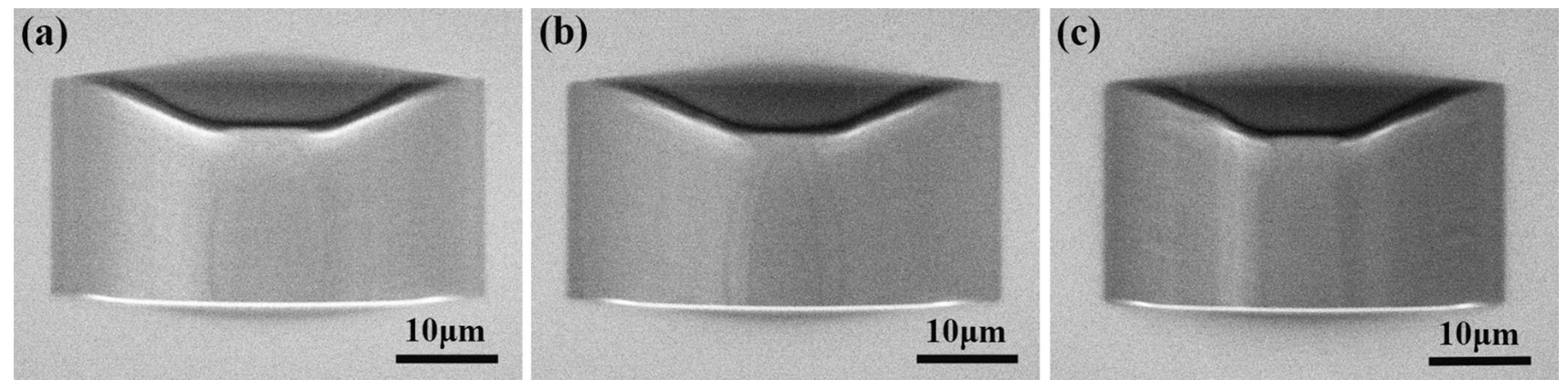

Fig. 16 The SEM images of the sinusoidal microstructures: a original bitmap milling; $\mathbf{b}$ in situ modification; and $\mathbf{c}$ iterative optimization

\section{(a)}

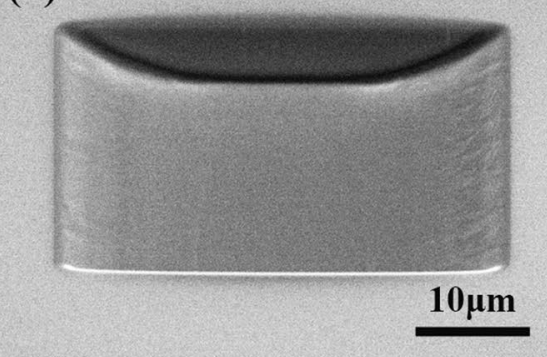

(b)

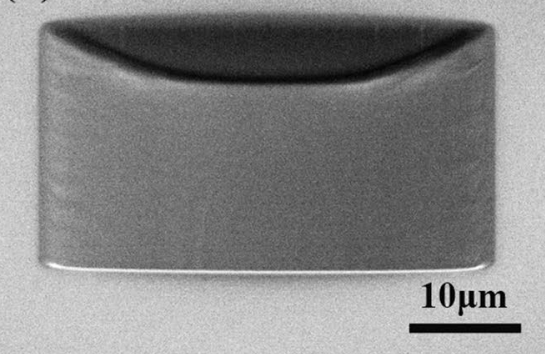

(c)

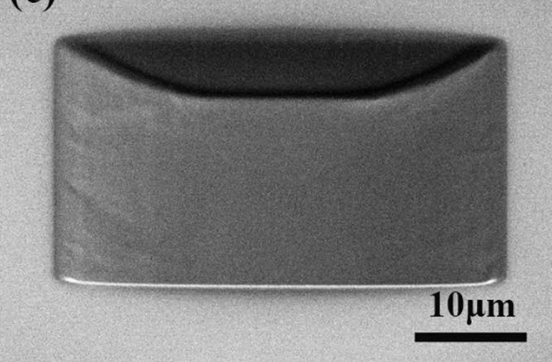

Fig. 17 The SEM images of concave cylindrical microstructures: a original bitmap milling; $\mathbf{b}$ in situ modification; and $\mathbf{c}$ iterative optimization 

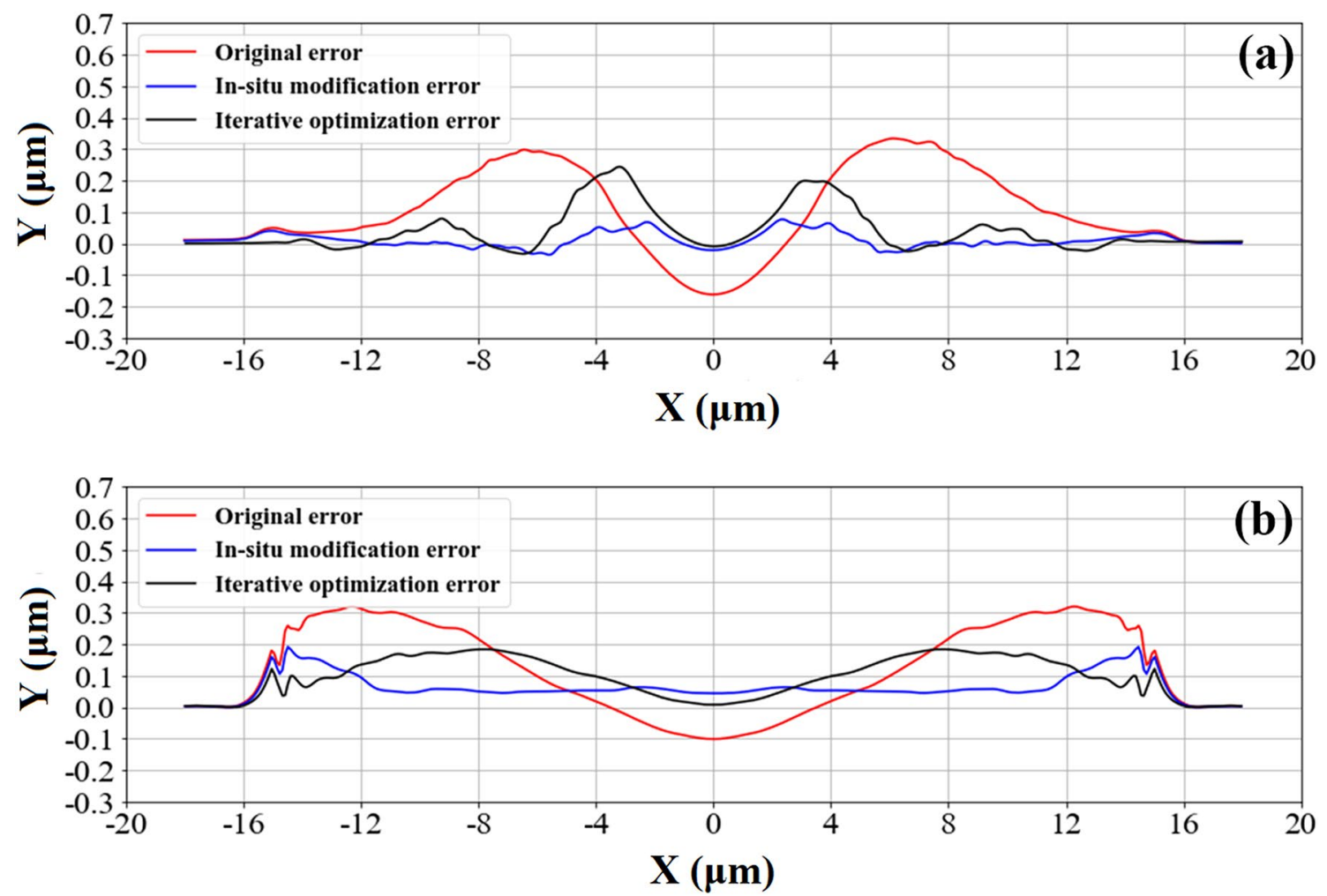

Fig. 18 The error curves of the microstructures milled using the original bitmap and the two compensation methods: a sinusoidal and $\mathbf{b}$ concave cylindrical

Table 1 Comparison of the shape accuracy improvement for the two error compensation methods

\begin{tabular}{lllll}
\hline Method & Shape accuracy & $\begin{array}{l}\text { Concave micro- } \\
\text { lens (\%) }\end{array}$ & Sinusoidal (\%) & $\begin{array}{l}\text { Concave } \\
\text { cylindrical } \\
(\%)\end{array}$ \\
\hline In situ modification & $P-V$ & 57 & 77 & 55 \\
& RMS & 56 & 85 & 59 \\
Iterative optimization & $P-V$ & 71 & 44 & 56 \\
& RMS & 54 & 53 & 40 \\
\hline
\end{tabular}

bitmap file, and thus, the actual processing of this method is more convenient.

\section{Conclusion}

The formation mechanism and compensation methods of the profile error in FIB bitmap milling of 3D optical microstructure are studied in this work. The influence of FIB milling parameters such as ion beam currents, dwell times, and beam overlap ratios on the profile error is investigated by the experimental measurement. A larger ion beam current improved the FIB milling efficiency, but it also led to the decrease in processing accuracy. A long dwell time causes the formation of residue at the bottom of the concave micro-lens due to the redepositing effect, which is detrimental for processing accuracy. The larger beam overlap ratio is favorable for the surface roughness; otherwise, there are beam move marks on the surface. The optimized parameters are 21 $\mathrm{nA}, 10 \mu \mathrm{s}$, and $50 \%$, and the shape accuracy processed by these parameters is $0.44 \mu \mathrm{m}$ of $P-V$ and $0.142 \mu \mathrm{m}$ of RMS, respectively. An accurate simulation model of the FIB milling profiles is used to investigate the intrinsic formation mechanism of the 3D profile error. By means of the simulation model, it can be found that the influence degree of ion beam diameters, redeposition effect, and sputter yields on the profile error increases successively, and these factors are inevitable in FIB milling of $3 \mathrm{D}$ microstructures. The in situ modification method and 
iterative optimization method are proposed to compensate the processing error, which can improve the shape accuracy more than $50 \%$. Although the iterative optimization method is more convenient as it only needs one bitmap file, it is not good at the enhancement of the global profiles error compared to the in situ modification method.

Acknowledgements This work was supported by the National Natural Science Foundation of China (Grant No. 51905195) and China Postdoctoral Science Foundation (Grant No. 2017M612447). The authors thank the Experiment Center of School of Mechanical Science and Engineering for the FIB experiments.

\section{Compliance with ethical standard}

Conflict of interest On behalf of all authors, the corresponding author states that there is no conflict of interest.

\section{References}

1. Khorasaninejad M, Capasso F (2017) Metalenses: versatile multifunctional photonic components. Science 358:1146. https:// doi.org/10.1126/science.aam8100

2. Chen WT, Zhu AY, Sanjeev V, Khorasaninejad M, Shi Z, Lee E, Capasso F (2018) A broadband achromatic metalens for focusing and imaging in the visible. Nat Nanotech 13:220-226. https ://doi.org/10.1038/s41565-017-0034-6

3. Khorasaninejad M, Chen WT, Devlin RC, Oh J, Zhu AY, Capasso F (2016) Metalenses at visible wavelengths: diffraction-limited focusing and subwavelength resolution imaging. Science 352(6290):1190-1194. https://doi.org/10.1126/science.aaf6644

4. Kim Y, Rhee H, Ghim Y, Lee $Y$ (2019) Method of fabricating an array of diffractive optical elements by using a direct laser lithography. Int J Adv Manuf Technol 101:1681-1685. https:// doi.org/10.1007/s00170-018-3058-7

5. Brousseau EB, Dimov SS, Pham DT (2010) Some recent advances in multi-material micro- and nano-manufacturing. Int J Adv Manuf Technol 47(1-4):161-180. https://doi.org/10.1007/s0017 0-009-2214-5

6. Shyu RF, Yang H (2006) Vacuum suction aid for microlens array formation using LIGA-like process. Int J Adv Manuf Technol 29(5-6):518-523. https://doi.org/10.1007/s00170-005-2549-5

7. Totsu K, Fujishiro K, Tanaka S, Esashi M (2006) Fabrication of three-dimensional microstructure using maskless gray-scale lithography. Sens Actuators A 130-131:387-392. https://doi. org/10.1016/j.sna.2005.12.008

8. Chen KS, Lin IK, Ko FH (2005) Fabrication of 3D polymer microstructures using electron beam lithography and nanoimprinting technologies. J Micromech Microeng 15(10):1894-1903. https ://doi.org/10.1088/0960-1317/15/10/015

9. Grigaliūnas V, Lazauskas A, Jucius D, Viržonis D, Abakevičienėa B, Smetonac S, Tamulevičiusa S (2016) Microlens fabrication by 3D electron beam lithography combined with thermal reflow technique. Microelectron Eng 164:23-29. https://doi.org/10.1016/j. mee.2016.07.003

10. Hasan M, Shahjahan I, Gopinadhan M (2018) Three-dimensional compatible sacrificial nanoimprint lithography for tuning the wettability of thermoplastic materials. J Micro Nano Manuf 6(4):041003. https://doi.org/10.1115/1.4041532
11. Tseng AA (2004) Recent developments in micromilling using focused ion beam technology. J Micromech Microeng 14(4):R15-R34. https://doi.org/10.1088/0960-1317/14/4/R01

12. De Felicis D, Mughal MZ, Bemporad E (2017) A method to improve the quality of 2.5 dimensional micro-and nanostructures produced by focused ion beam machining. Micron 101:8-15. https://doi.org/10.1016/j.micron.2017.05.005

13. Rommel $M$, Jambreck JD, Ebm C, Platzgummer E, Bauer AJ, Frey $L$ (2010) Influence of FIB patterning strategies on the shape of 3D structures: comparison of experiments with simulations. Microelectron Eng 87(5-8):1566-1568. https://doi. org/10.1016/j.mee.2009.10.054

14. Bilbao-Guillerna A, Eachambadi RT, Cadot GBJ, Axinte DA, Billingham J, Stumpf F, Beuer S, Rommel M (2018) Novel approach based on continuous trench modelling to predict focused ion beam prepared freeform surfaces. J Mater Proces Technol 252:636-642. https://doi.org/10.1016/j.jmatprotec .2017.10.024

15. Kim CS, Ahn SH, Jang DY (2012) Review: developments in micro/nanoscale fabrication by focused ion beams. Vacuum 86(8):1014-1035. https://doi.org/10.1016/j.vacuum.2011.11.004

16. Vasile MJ, Nassar R, Xie J, Guo H (1999) Microfabrication techniques using focused ion beams and emergent applications. Micron 30(3):235-244. https://doi.org/10.1016/S0968 -4328(99)00008-6

17. Fu Y, Kok N, Bryan A (2000) Microfabrication of microlens array by focused ion beam technology. Microelectron Eng 54(34):211-221. https://doi.org/10.1016/S0167-9317(00)00416-0

18. Fu Y, Bryan NKA (2000) Investigation of $3 D$ microfabrication characteristics by focused ion beam technology in silicon. J Mater Proces Technol 104(1-2):44-47. https://doi.org/10.1016/ S0924-0136(00)00544-6

19. Vasile MJ, Niu Z, Nassar R, Zhang W, Liu S (1997) Focused ion beam milling: depth control for three-dimensional microfabrication. J Vac Sci Technol B 15(6):2350-2354. https://doi. org/10.1116/1.589644

20. Fang FZ, Xu ZW, Hu XT, Wang CT, Luo XG, Fu YQ (2010) Nanophotomask fabrication using focused ion beam direct writing. CIRP Ann Manuf Technol 59:543-546. https://doi.org/10.1016/j. cirp.2010.03.038

21. Vayalamkuzhi P, Bhattacharya S, Eigenthaler U, Keskinbora K, Samlan CT, Hirscher M, Spatz JP, Viswanathan NK (2016) Direct patterning of vortex generators on a fiber tip using a focused ion beam. Opt Lett 41(10):2133. https://doi.org/10.1364/ OL.41.002133

22. Niessen F, Nancarrow MJB (2019) Computer-aided manufacturing and focused ion beam technology enable machining of complex micro- and nano-structures. Nanotechnology 30:43. https://doi.org/10.1088/1361-6528/ab329d

23. Chiu Y, Huang C H, Hsu Y C (2007) High numerical-aperture microlens fabricated by focused ion beam milling. In: Proceedings of SPIE - the international society for optical engineering 6620. https://doi.org/10.1117/12.738639

24. Sun J, Luo X, Ritchie J, Hrncir T (2012) A predictive divergence compensation approach for the fabrication of three-dimensional microstructures using focused ion beam machining. Proc Inst Mech Eng B J Eng Manuf 226(2):229-238. https://doi. org/10.1177/0954405411408185

25. Sun J, Luo X, Ritchie JM, Chang W, Wang W (2012) An investigation of redeposition effect for deterministic fabrication of nanodots by focused ion beam. Precis Eng 36(1):31-36. https://doi. org/10.1016/j.precisioneng.2011.07.001

26. Fu YQ, Bryan NKA, Shing ON, Hung NP (2000) Influence of the redeposition effect for focused ion beam 3D micromachining in silicon. Int J Adv Manuf Technol 16:877-880. https://doi. org/10.1007/s001700070005 
27. Lindsey S, Waid S, Hobler G, Wanzenböck HD, Bertagnolli E (2014) Inverse modeling of FIB milling by dose profile optimization. Nucl Instrum Methods Phys Res B 341(2014):77-83. https ://doi.org/10.1016/j.nimb.2014.09.006

28. Kim HB (2012) Multiple surface driving method for the accurate sculpting of predefined arbitrary surfaces in the micro/ nano regime. Microelectron Eng 91(2012):14-18. https://doi. org/10.1016/j.mee.2011.11.015

29. Kim HB, Hobler G, Steiger A, Lugstein A, Bertagnolli E (2007) Full three-dimensional simulation of focused ion beam micro/ nanofabrication. Nanotechnology 18(24):245303. https://doi. org/10.1088/0957-4484/18/24/245303

30. Kim HB, Hobler G, Lugstein A, Bertagnolli E (2007) Simulation of ion beam induced micro/nano fabrication. J Micromech Microeng 17(6):1178-1183. https:// doi.org/10.1088/0960-1317/17/6/011

31. Yamamura Y, Itikawa Y, Itoh N (1983) Angular dependence of sputtering yields of monatomic solids. IPPJ-AM reports, Nagoya, Japan Institute of Plasma Physics, Nagoya Univ

Publisher's Note Springer Nature remains neutral with regard to jurisdictional claims in published maps and institutional affiliations. 